\title{
EDITORIAL
}

\section{Myocardial infarction, peptic ulcer and acetylsalicylic acid: Of good and evil}

\author{
John K Marshall MD MSc FRCPC AGAF
}

$\mathrm{D}_{\mathrm{s}}^{\mathrm{s}}$ Jekyll famously noted that all men are 'comingled with good and evil'. So too, are most medical interventions. Acetylsalicylic acid (ASA) has a proven benefit in the treatment of myocardial infarction, and for primary and secondary cardiovascular prophylaxis. It may even prevent cancer. However, ASA is equally infamous for its ability to injure the gastrointestinal tract, causing complicated peptic ulcer disease. As evidence of their cardiovascular benefit continues to accrue, anticoagulant and antiplatelet therapies will be prescribed more often and therapeutic endoscopists will be kept busy. Gastroenterologists who manage the complications of ASA-associated gastropathy are understandably keen to prevent its recurrence and often recommend that ASA be either held or withdrawn. While it is intuitively sensible that avoiding ASA will minimize the risk of rebleeding, this may not be of net clinical benefit when all the consequences of that decision are considered.

In the present issue of The Canadian Journal of Gastroenterology, Cheung et al (pages 619-623) (1) retrospectively reviewed data from 102 patients admitted to hospital with acute myocardial infarction who then developed a bleeding peptic ulcer. All patients received proton pump inhibitors (PPIs) and almost all with high-risk ulcer stigmata received endoscopic therapy. In most cases (63\%), ASA was discontinued. Individuals who were continued on ASA were less likely to have high-risk ulcer stigmata on endoscopy and more likely to have required coronary intervention. Patients continuing versus stopping ASA did not differ significantly in 30-day rebleeding rates, mortality or length of stay.

This small study profiled clinical behaviour at two tertiary care centres and, as such, may not be generalizable to other practice settings. Reassuringly, it demonstrates that clinicians made rational decisions to selectively continue ASA in patients with low gastrointestinal risk and high cardiovascular risk. More importantly, however, it shines light on a common but high-stakes clinical scenario, and poses two fundamental questions about the clinical consequences of stopping or continuing ASA.

First, does continuing ASA impair ulcer healing or increase the risk of rebleeding? All patients recovering from an acute ulcer bleed should receive a PPI. Randomized trials have demonstrated that combining low-dose ASA with a PPI reduces the risk of ulcer complications versus either clopidogrel monotherapy $(2,3)$ or ASA monotherapy (4), following an ASAinduced peptic ulcer bleed. Another trial (5), which enrolled only low-risk patients, showed the rate of endoscopic injury to be low in subjects given either low-dose ASA or clopidogrel who received a PPI after recovering from ASA-induced peptic ulcer disease. However, these landmark trials all fall short of answering our question for two reasons:

1. All were conducted in Asian populations (in which PPIs are considered to be more effective); and
2. Each protocol called for ASA to be stopped and reintroduced only after the index ulcer had healed.

There are abundant data to suggest that PPI therapy can heal ulcers in patients who continue to receive conventional nonsteroidal anti-inflammatory drugs, but healing is not universal and such a strategy has not been tested rigorously in high-risk patients receiving ASA.

A second question is whether stopping ASA leads to adverse cardiovascular outcomes. Sung et al (6) unequivocally demonstrated this in a small randomized trial among patients treated for ASA-associated peptic ulcer bleeding. Stopping ASA therapy did not reduce rebleeding, but was associated with a clinically significant increase in overall mortality. This concept was reinforced by a large meta-analysis (7) that associated ASA withdrawal (for any reason) with an increased risk of major adverse coronary events, particularly among patients with intracoronary stents. When an ulcer begins to bleed after a myocardial infarction, there may well be an optimal interval for which ASA can be withdrawn to facilitate healing without compromising cardiovascular outcomes; this, however, remains to be defined.

Ultimately, these questions will only be answered with adequately powered and well-controlled clinical trials. Until such data are available, clinicians must continue to weigh the pros and cons of alternative approaches, and individualize risk assessments. Perhaps the greatest lesson is that neither gastrointestinal nor cardiovascular risk should be considered in isolation. When charmed by Dr Jekyll, always stay mindful of Mr Hyde.

\section{REFERENCES}

1. J Cheung, J Rajala, D Moroz, Q Zhu, M Stamm, GS Sandha. Acetylsalicylic acid use in patients with acute myocardial infarction and peptic ulcer bleeding. Can J Gastroenterol 2009;23:619-23.

2. Chan FK, Ching JY, Hung LC, et al. Clopidogrel versus aspirin and esomeprazole to prevent recurrent ulcer bleeding. N Engl J Med 2005;352:238-44.

3. Lai KC, Chu KM, Hui WM, et al. Esomeprazole with aspirin versus clopidogrel for prevention of recurrent gastrointestinal ulcer complications. Clin Gastroenterol Hepatol 2006;4:860-5.

4. Lai KC, Lam SK, Chu KM, et al. Lansoprazole for the prevention of recurrence of ulcer complications from long-term low-dose aspirin use. N Engl J Med 2002;346:2033-8.

5. Ng FH, Wong BC, Wong SY, et al. Clopidogrel plus omeprazole compared with aspirin plus omeprazole for aspirin-induced symptomatic peptic ulcers/erosions with low to moderate re-bleeding risk: A single-blind randomized controlled trial. Aliment Pharmacol Ther 2004;19:359-65.

6. Sung J, Lau J, Ching J, et al. Early reintroduction of aspirin with proton pump inhibitor after endoscopic hemostasis for peptic ulcer bleeding: Final results of a double blinded randomized study. Gut 2007;56:A27. (Abst)

7. Biondi-Zoccai GGL, Lotrionte M, Agostoni P, et al. A systematic review and meta-analysis on the hazards of discontinuing or not adhering to aspirin among 50,279 patients at risk for coronary disease. Eur Heart J 2006;27:2667-74.

Division of Gastroenterology, McMaster University Medical Centre, Hamilton, Ontario

Correspondence: Dr John K Marshall, Division of Gastroenterology (2F59), McMaster University Medical Centre, 1200 Main Street West,

Hamilton, Ontario L8N 325. Telephone 905-521-2100 ext 76782, fax 905-523-6048, e-mail marshllj@mcmaster.ca

Received and accepted for publication June 9, 2009 


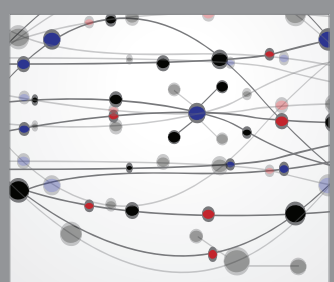

The Scientific World Journal
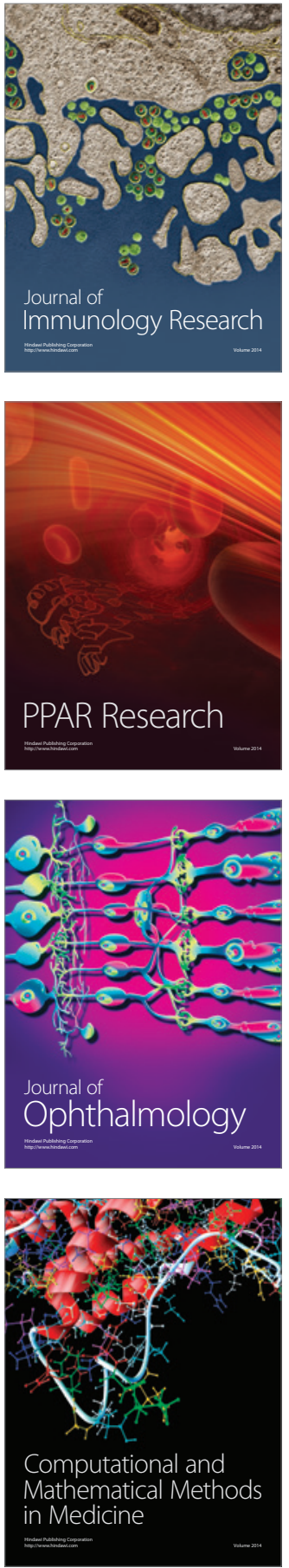

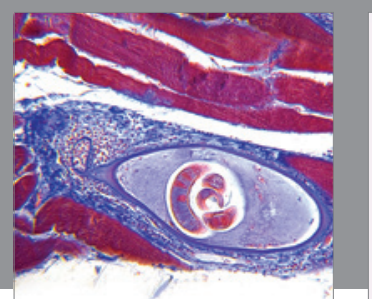

Gastroenterology Research and Practice

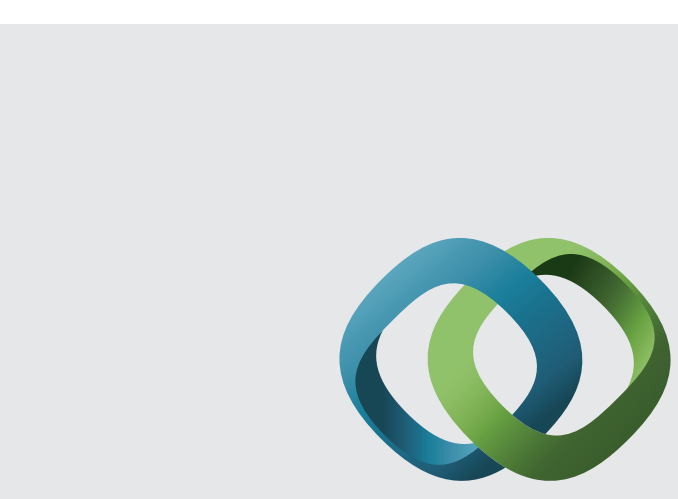

\section{Hindawi}

Submit your manuscripts at

http://www.hindawi.com
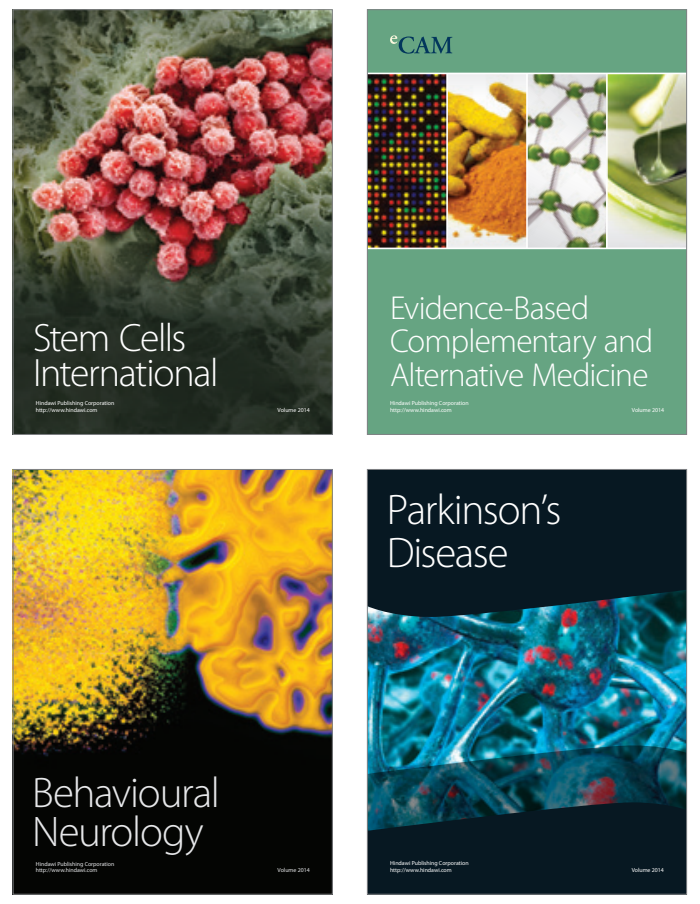
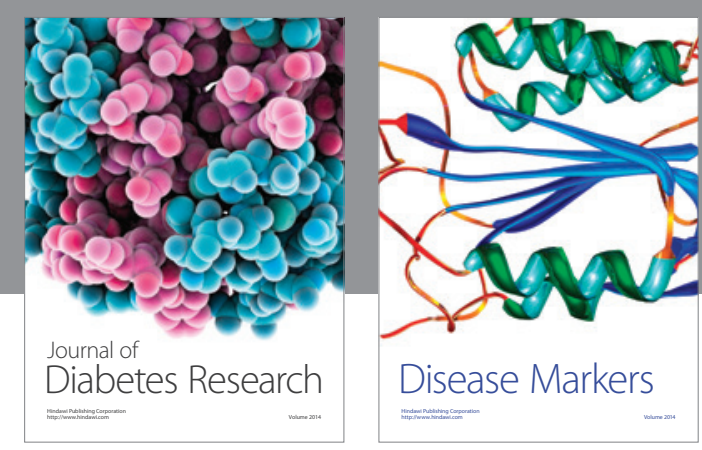

Disease Markers
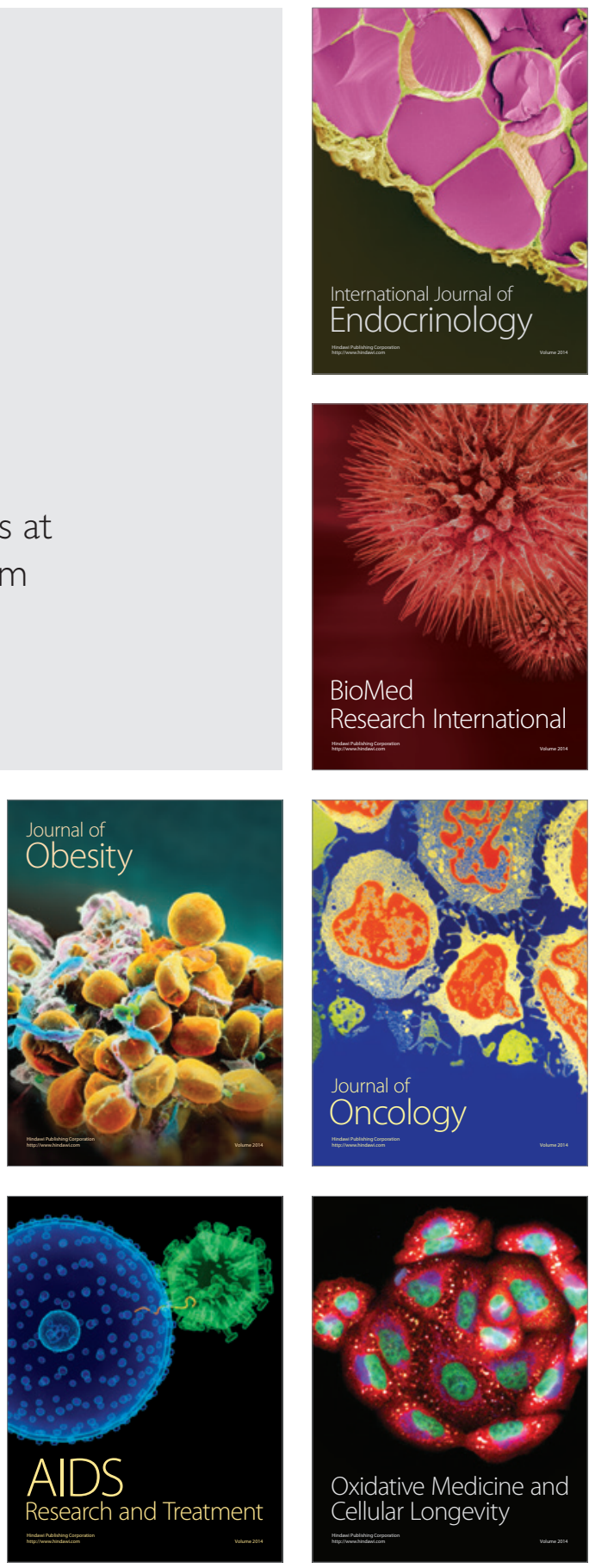